\title{
CONOCIMIENTOS CULTURALES COMO CONTENIDOS DE LA EDUCACIÓN FAMILIAR MAPUCHE ${ }^{\mathbf{1}}$
} Cultural knowledge as a content of mapuche family education

Segundo Quintriqueo M.* Daniel Quilaqueo R.** Fernando Peña-Cortés***

Gerardo Muñoz T.****

\section{Resumen}

El artículo tiene por objeto analizar la construcción del conocimiento mapuche según el discurso de kimches. Sostenemos que en la educación familiar existe un proceso de construcción de conocimientos propios como un sistema de saberes y contenidos educativos para la formación de personas. La metodología empleada es la investigación educativa. Los resultados parciales muestran una descripción acerca de la lógica de los conocimientos educativos propios, para contextualizar la enseñanza y el aprendizaje de las ciencias en el medio escolar, desde la educación intercultural en contexto mapuche.

Palabras clave: Epistemología, Conocimiento cultural mapuche, Educación familiar mapuche, Educación intercultural.

\section{Abstract}

The aim of this article is to analyze the construction of Mapuche knowledge based on the kimches speech. We argue that within family education, there exists a process of constructing their own knowledge, as a system of knowledge and educational contents for forming individuals. The research methodology applied corresponds to education research. Preliminary results describe the logic of individual educational knowledge from intercultural education in the Mapuche context, so as to contextualize the teaching and learning of Science in schools.

Key words: Epistemology, Mapuche cultural knowledge, Mapuche family education, Intercultural education.

\section{INTRODUCCIÓN}

El contexto de estudio de la investigación es el borde costero de La Araucanía, delimitado por la cordillera de la Costa y las planicies litorales. En la perspectiva de Peña-Cortés, Almendra y Rojas (2010) el borde costero contiene una amplia variedad de paisajes y biodiversidad, donde se destaca la formación de ecosistemas de humedales capaces de contener y propiciar el desarrollo de vida microorgánica,

\footnotetext{
${ }^{1}$ El artículo informa los resultados de Proyectos financiados por el Fondo Nacional de Desarrollo Científico y Tecnológico (FONDECYT) REGULAR Nº 1110489 y 1140490.
} 
vegetal y animal. En la región de La Araucanía, el borde costero está comprendido por las comunas de Carahue, Saavedra, Teodoro Schmidt y Toltén, las que presentan una alta población mapuche organizada en familias y comunidades con identidad lafkenche — gente que vive a orillas del mar-(Peña-Cortés et al., 2010).

En la investigación sostenemos que en La Araucanía existe una relación hegemónica del conocimiento escolar acerca del mapuche, lo que genera una distancia epistemológica entre ambos y obstaculiza el aprendizaje intercultural (Quilaqueo, Fernández y Quintriqueo, 2010). Además, obstaculiza la interacción y relación social entre estudiantes mapuches y no mapuches, y de los estudiantes con sus profesores, producto de un proceso de escolarización monocultural.

El objetivo del artículo es analizar la construcción del conocimiento cultural mapuche en el marco de la educación familiar como contenidos educativos deseables de incorporar en los procesos educativos interculturales.

\section{MARCO DE REFERENCIA}

Desde la tradición histórica republicana, el currículum escolar chileno es monocultural. En este sentido, la naturaleza de la escuela se relaciona con la transmisión de un patrimonio cultural único a las nuevas generaciones, con la intención de atenuar las diferencias y desigualdades sociales, culturales, educacionales y económicas (Quintriqueo, 2010). Sin embargo, en América Latina y especialmente en Chile, las desigualdades y las diferencias son producto de procesos de escolarización (Baudelot y Leclerq, 2008). Al respecto, la educación escolar ha desconocido los saberes construidos por las familias y comunidades indígenas, negando con ello la existencia de una racionalidad en su forma de conocer y comprender el medio natural, social, cultural y espiritual. En esta lógica de formación escolar los alumnos, padres y los propios profesores asumen la escuela como un espacio y una institución legitimada social y legalmente para transmitir el saber y el conocimiento occidental (Rockwell, 1995; 2008; Quintriqueo, Quilaqueo y Torres, 2014). Así, en el caso de La Araucanía se construye una representación respecto de 'lo mapuche' y especialmente una hegemonía del conocimiento occidental por sobre el conocimiento cultural mapuche, donde no se acepta otra lógica que no sea la occidental.

Por consiguiente, la epistemología del conocimiento occidental, base del conocimiento escolar, se fundamenta en los siguientes supuestos: 1) Asume que todo acto de conocimiento se realiza en la relación sujeto-objeto, donde la dualidad es aceptada como un elemento necesario; 2) Para que exista el conocimiento, algo es objeto de conocimiento para un sujeto cognoscente, donde el sujeto transciende el polo de la subjetividad; y 3) La trascendencia de la subjetividad forma una imagen del objeto en el sujeto como una representación abstracta. En este proceso, el sujeto puede determinar al objeto como imagen que posee de él para construir una noción de verdad (Izquierdo, 1998; Quintriqueo y Cárdenas, 2010). 
Así, la racionalidad monocultural del conocimiento occidental se fundamenta en los siguientes postulados: 1) La realidad existe independientemente de las representaciones humanas, mentales y lingüísticas; 2) La realidad existe independientemente del lenguaje, donde se asume que la lengua sirve principalmente para transmitir información; 3) La verdad es un asunto de precisión sobre la representación, donde la afirmación de la verdad está asociada a la perfección como un aspecto mejorable, pero no depende de un punto de vista o de una perspectiva subjetiva; 4) El conocimiento es objetivo e independiente de los sujetos; 5) Hay una reducción del sujeto a sus funciones intelectuales y cognitivas, donde las creencias y la subjetividad son asuntos secundarios; 6) Los factores culturales y simbólicos de la vida colectiva son desvalorizados, los que se asumen como fenómenos fácilmente observables, por lo que escapan a la dimensión racional y cognitiva de las personas; 7) Se centra en la creencia de una base biológica del comportamiento para explicar las actitudes y los comportamientos de las personas; y 8) Se muestra principalmente las imágenes de los éxitos del pensamiento occidental, como un ideal de progreso continuo y del desarrollo en la evolución humana (Alsina, 1999; Ardoino, 2005; García, 2007; Quintriqueo y Cárdenas, 2010).

Por su parte, la literatura científica hoy propone una epistemología contextual e intercultural definida como una contraposición a la racionalidad monocultural y como un desafio de abrir nuevas formas de comprender y explicar la construcción de conocimientos (Ardoino, 2005; Castellano y Hinestroza, 2009; Fourez, 2009). La epistemología contextual, en contexto mapuche, es un estudio interdisciplinario emergente, que sin descartar otras posturas epistemológicas devela los problemas del conocer, del saber y los conceptos relacionados con el sentido y la inteligencia, para comprender la realidad sociocultural (Nadeau, 2009). Es una epistemología donde las palabras no tienen realidad en sí mismas, sino que se les atribuyen una valoración respecto de su pertenencia a la memoria individual y social. Las palabras no tienen sentido si no hay personas que las piensen, siendo por tanto producto de una construcción social (Quintriqueo y Torres, 2013). En este sentido, actualmente es posible encontrar epistemologías diversas, concurrentes, contradictorias y diferentes, que podrían contribuir para estudiar y comprender la complejidad de hacer ciencias en contextos de interculturalidad (Jamioy, 1997; Ramírez, 2001; Ardoino, 2005; Cyrulink y Morin, 2005; Quintriqueo, Quilaqueo y Torres, 2014). Es un proceso que se basa en la relación de saberes originarios y occidentales, como respuesta al agotamiento progresivo de la racionalidad instrumental (Castellano y Hinestroza, 2009; Olivé, 2009; Quintriqueo y Torres, 2012).

En esa perspectiva, la epistemología contextual e intercultural se fundamenta en los siguientes supuestos: 1) La realidad es una construcción social que no existe independiente de los actores, del contexto y las teorías implícitas que le dan forma, donde la lengua permite conceptualizarla y comunicarla desde una lógica propia; 2) Toda objetividad es objetividad a partir de una versión más o menos eficaz para 
comprender la realidad; 3) Las interpretaciones son subjetivas sobre la base de una serie de enunciados, cuyo sentido y estatus referencial están asociados a explicaciones de la realidad sociocultural y las posiciones de los sujetos en los actos individuales y sociales, en un contexto específico; 4) Los valores y las verdades son relativas, asociadas a la historia individual y social de los sujetos, lo que obliga a relativizar todo juicio de valor; y 5) El conocimiento es un hecho político, porque las categorías y los valores sociales son el resultado de actividades sociales, construidos en relaciones directas y concretas con el medio natural, social, cultural y espiritual (Filippa y Schiattino, 1997; Ardoino, 2005; Quintriqueo y Torres, 2012).

Desde la epistemología contextual e intercultural, el pensar reductivo, polarizante, absolutista y/o simplista de la escuela no se puede comprender sin considerar la complejidad de la realidad, del mundo actual en el campo educativo (Alsina, 1999). En la actualidad, el conocimiento científico es relativo, donde no hay verdades absolutas ni las incertidumbres del conocimiento cotidiano son completas. Entonces, desde una perspectiva comprensiva y dialógica del currículum se abandona la antigua concepción del conocimiento único y apuesta a la necesidad de interrogarlo y comprenderlo desde su contexto. Así, estudiar el conocimiento de la escuela tiene que ver con exigencias que comprendemos como vías a transitar y un proyecto a construir, para generar la relación de saberes en los estudiantes, lo que les permita comprender la realidad objetiva y subjetiva (López y Merleau-Ponty, 2008; Quintriqueo y McGinity, 2009). En este sentido, la educación escolar no puede desconocer las singularidades de los individuos como sujetos que construyen la historia, que actúan en relación con los hechos sociohistóricos y que el conocimiento nunca deja de construirse (Mutuale, 2009). Entonces, el sentido del saber, lejos de fijarse en lo puramente objetivo o subjetivo, se configura mediante procesos en espirales recursivas y en la relación de saberes. Gestar y validar el conocimiento nos remite a la presencia de la ciencia y del lenguaje en la escuela, a su comprensión y a los modos legítimos de relación con el conocimiento cotidiano (Rockwell, 1995; Rodrigo y Arnay, 1997; García, 2007). Todo conocimiento científico y cotidiano supone construcciones y no puede avanzar ajeno a la experiencia. Por su parte, el estudiante al ingresar a la escuela es portador de un conjunto de conocimientos y visiones de la realidad, lo que pertenece a su contexto cotidiano, cultural y científico (Quintriqueo y McGinity, 2009). El supuesto es que los profesores y adultos reconocen que los estudiantes saben y que ellos reconocen sus propios conocimientos para el aprendizaje de las ciencias, partiendo de lo que se sabe, modificando, cuestionando, incluso reafirmando el conocimiento de la educación familiar (García, 2007).

La educación familiar se define como la acción educativa donde los niños y adolescentes se disponen para el aprendizaje de contenidos educativos que se fundamentan en las experiencias de relación social del grupo (Beillerot y Mosconi, 2006). En este sentido, la noción de educación familiar comprende el conjunto de 
intervenciones sociales organizadas para preparar, sostener, ayudar y formar a las nuevas generaciones. Así, los procesos educativos constituyen un objetivo complejo realizado en el contexto familiar, ya sea en forma interna o bien como intervenciones socioeducativas con el objeto de revitalizar la memoria individual y social de la comunidad, respecto de saberes y conocimientos educativos (Beillerot y Mosconi, 2006). Entonces, la educación familiar hoy tiene relevancia en el marco de la participación y toma de decisión en la familia y la comunidad, para avanzar hacia una educación intercultural.

La educación con enfoque intercultural es una alternativa que permite reconocer la diversidad de saberes y conocimientos que convergen en la escuela, donde la construcción de contenidos educativos está sustentada de manera epistemológica en la racionalidad del conocimiento mapuche, campesino o popular, lo que generaría una educación contextualizada social, cultural, natural y espiritual, pertinente a la realidad de los estudiantes (Quintriqueo y Torres, 2012).

\section{METODOLOGÍA}

La metodología empleada es la investigación educativa, con el objeto de construir una base de conocimientos para comprender y mejorar la educación en contextos interculturales, basado en el enfoque multimétodo (Gauthier Clermont et al., 1997; Bisquerra, 2004; Ruiz, 2008). El enfoque multimétodo puede ser entendido como una estrategia donde se utilizan dos o más procedimientos para la indagación en un mismo fenómeno u objeto de estudio, mediante los diferentes momentos acerca del proceso de investigación. La producción de información cuantificable y descriptiva permite una comprensión y explicación del objeto de estudio, lo que no sería posible lograr utilizando los métodos cualitativo y cuantitativo por separado (Tashakkori y Teddlie, 2003; Ruiz, 2008). Su utilización se justifica en que el interés de relacionarla directamente con la educación, para analizar la complejidad que presenta la relación entre el saber mapuche y el conocimiento escolar. Otros enfoques metodológicos retenidos son: 1) La investigación socio-educacional (Bonal, 1998; Gauthier Benoît, 2009), que centra su interés en estudiar problemas ligados a la educación, en cuyo proceso la comprensión del fenómeno educativo se realiza con los aportes de la pedagogía y la sociología de la educación; y 2) El análisis socio histórico (Durán y Cárdenas, 1983; Egaña, 2000), que centra su interés en el fenómeno educativo desde una retrospectiva para comprender la educación, considerando el contexto histórico, fundamentalmente desde la institucionalidad, los procesos políticos, curriculares y socioculturales de la realidad regional, nacional e internacional.

La investigación se inscribe en el contexto de escuelas situadas en comunidades mapuches rurales y semirrurales de la territorialidad Lafkenche — borde costero- de la región de La Araucanía (Figura 1), con matrícula de alumnos mapuches y no mapuches. El estudio considera una muestra total de 24 kimches (sabios). La técnica de muestreo empleada es intencionada, con una selección de 
casos típicos, basada en los criterios de edad, pertenencia mapuche, género y áreas geográficas. El procesamiento y análisis de los datos (entrevistas, discursos, registros etnográficos) se realiza mediante el análisis de contenido (Krippendorff, 1990; Quivy R. y Campenhoudt, 1998) apoyándose en el software Atlas.ti 5.0, para hacer más eficiente y fluido el procesamiento y presentación de la información. El análisis de contenido busca develar núcleos de saberes centrales de orden abstracto, dotados de sentido, desde la perspectiva de los productores del discurso, a partir de contenidos concretos expresados en el texto y contenidos latentes (Pérez, 2000).

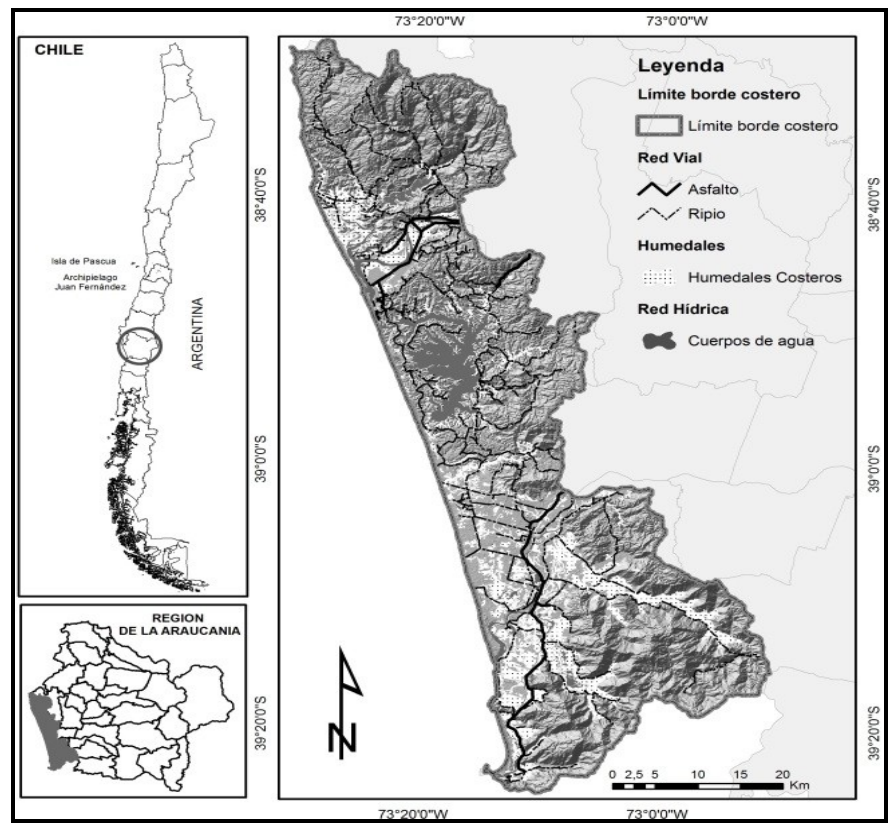

Figura 1. Borde costero, Región de La Araucanía, Chile.

\section{RESULTADOS}

Los resultados se refieren a una categoría de la investigación Fondecyt $\mathrm{N}^{\circ}$ 1110489 , referida a saberes y conocimientos mapuches. En relación con dicha categoría constatamos seis dimensiones: a) Conocimientos acerca de la historia local; b) Conocimientos relativos al parentesco y su territorio; c) Prácticas socioculturales y costumbres mapuches; d) Conocimiento referente al medio natural y sobrenatural; e) Conocimiento respecto del cultivo agrícola; y f) Saberes educativos mapuches (ver Tabla 1). Al analizar los porcentajes de recurrencia del discurso de los kimches acerca de estas dimensiones, constatamos que el conocimiento del parentesco y su territorio alcanza el 22,6\% de 633 recurrencias, asociado al origen paterno, materno, y al espacio en el que se desarrollan. En tanto, las prácticas socioculturales y las costumbres mapuches llegan a 22,6\%. Ambas dimensiones se observan como 
contenidos y finalidades educativas centrales para la formación de la identidad sociocultural de las personas, desde la educación familiar mapuche, lo que en su conjunto alcanza el 45,2\% de las recurrencias. Las dimensiones conocimiento referente a la historia local y conocimientos del medio natural y espiritual configuran un segundo grupo alcanzando un $38,7 \%$. Estos saberes se relacionan directamente con valores educativos que son aplicables al respeto y el cultivo del aprecio, tanto en las relaciones entre las personas como con el medio natural y las fuerzas espirituales que fundamentan la cosmovisión mapuche. Un tercer grupo de dimensiones del saber, se relaciona con el cultivo agrícola y saberes educativos mapuches alcanzando un $16,1 \%$. En relación con estos saberes se constata que tienen una baja frecuencia y es probable que se vinculen, por una parte, con un progresivo aumento de suelos no cultivables, producto de la sobreexplotación y división de las reducciones. Por otra parte, la pérdida de la enseñanza intencionada del saber mapuche en el medio familiar podría ser producto del aumento sistemático de procesos de escolarización con ausencia del conocimiento propio de los estudiantes de ascendencia mapuche del borde costero.

Tabla 1: Dimensiones del saber y conocimiento mapuche

\begin{tabular}{|l|c|c|}
\hline \multicolumn{1}{|c|}{ CÓDIGO } & FRECUENCIA & $\%$ \\
\hline $\begin{array}{l}\text { Conocimientos relativos al parentesco y su } \\
\text { territorio }\end{array}$ & 143 & 22,6 \\
\hline Prácticas socioculturales y costumbres mapuches & 143 & 22,6 \\
\hline Conocimiento del medio natural y espiritual & 132 & 20,9 \\
\hline Conocimiento acerca de la historia local & 113 & 17,8 \\
\hline Saberes educativos mapuche & 62 & 9,8 \\
\hline Conocimientos respecto del cultivo agrícola & 40 & 6,3 \\
\hline TOTAL & 633 & 100,0 \\
\hline
\end{tabular}

A continuación analizamos la educación familiar asociada a la historia local, la valoración del parentesco, el territorio y las prácticas socioculturales como contenidos centrales.

\subsection{CONOCIMIENTOS SOBRE LA HISTORIA LOCAL}

El conocimiento acerca de la historia local se relaciona con los siguientes procesos sociales: 1) El asentamiento ancestral de las familias mapuches en un lugar, el que más tarde es formalizado por el proceso de radicación de comunidades; 2) Los hechos que han impulsado los procesos migratorios internos, por medio del matrimonio entre personas de diferentes comunidades, ello implica el traslado de la mujer a la familia del hombre o viceversa; 3) La participación de los antepasados en la Guerra de Arauco, esto implicó la llegada o salida de miembros y familias de la comunidad; y 4) Los cambios en el sistema de vida de los individuos por la 
transformación de la geografía local y por catástrofes naturales, en especial el desplazamiento de familias y comunidades producto del maremoto de 1960.

Los hechos sociales y procesos históricos que permitieron el asentamiento de familias y comunidades en la isla Wapi se vincula con el tipo de relación establecida con el medio natural, social, cultural y espiritual. En los testimonios se constata que la migración de las familias hacia la isla Wapi, ubicada en medio del cuerpo de agua del lago Budi y el océano Pacífico, fue motivada por las características geográficas y la amplia biodiversidad existente. Al respecto un kimche señala: "[...] los Caripan, Aillapan; Hechunpan y Huilipan fueron los primeros en llegar a Wapi, quienes eran hermanos. Yo conocí a un difunto anciano llamado Guillermo Aillapan, el que siempre le decía primo a mi padre, y yo nunca había logrado comprender por qué [...]" (S3, P9). En el testimonio se constata que el asentamiento ancestral se desarrollaba por la familia ampliada. Así, es posible inducir que cada uno de los hermanos mencionados en el testimonio llevaba consigo a su esposa, hijos y nietos, permaneciendo en su conjunto como una comunidad unida en torno a un tronco parental común.

Un aspecto importante que expresan los testimonios es la ocupación de la isla por personas no mapuches, con lo que comienza una relación de coexistencia. En el caso particular de la isla Wapi, un kimche señala: "[...] Entonces, el anükon - hombre que vive en la comunidad de su esposa - les dijo a los Díaz que se escondieran por aquí, en un lugar aledaño al río Budi. Posteriormente, esos mismos wigkas trajeron a otros chilenos a vivir acá [...]" (S3, P81). Así, los nexos de relación familiar, el mestizaje y la amistad fueron motivos centrales para aceptar el ingreso de personas no mapuches a la comunidad.

Para las comunidades lafkenches el maremoto de 1960 constituye un proceso significativo de la historia local, ya que expresa la relación hombre-naturaleza que tradicionalmente se ha establecido. Antes del maremoto los cerros estaban cubiertos de una densa vegetación nativa. Actualmente es el lugar donde se ha construido el camino público y son espacios geográficos utilizados para la agricultura y ganadería, producto de ello se observa una erosión progresiva del suelo y contaminación del lago, lo que se fundamenta en la constante crecida del lago, inundando los antiguos caminos y terrenos agrícolas. En este proceso desaparecen en forma acelerada los bosques nativos, y al mismo tiempo se observa un aumento de la forestación con árboles exóticos como pino y eucaliptos, los que han impactado en la sequía de las napas subterráneas y han erosionado significativamente el suelo.

Un testimonio señala: "Entonces el maremoto cubrió grandes extensiones de terreno dedicado al pastoreo, a las siembras y el camino central de Nawel Wapi (isla donde habita el jaguar), quedaron cubiertas por el agua, hasta los cerros. Aquí cuando baja el lago en la boca del Budi, está tapado con junquillo y eso no sirve ni para los animales" (S2, P35). En el testimonio, la expresión Nawel hace referencia a un felino similar al jaguar, actualmente extinto. Se constata además que el maremoto provocó, 
en gran parte del borde costero de La Araucanía, una inundación de terrenos cultivables o destinados a la ganadería. Al mismo tiempo, la inundación generó un movimiento migratorio de las familias hacia los terrenos altos, espacios actualmente sobreexplotados y, en consecuencia, se generó un cambio significativo en la geografía y el territorio Lafkenche.

\subsection{CONOCIMIENTOS RELATIVOS AL PARENTESCO Y EL TERRITORIO}

En el conocimiento educativo mapuche, el parentesco está asociado a un territorio en el que se desarrolla una complementariedad del küpan - ascendencia familiar vinculada a un territorio - y tuwün —origen territorial asociado a la familia de procedencia-. Así, el parentesco considera un conjunto de saberes y conocimientos que son conocidos por las nuevas generaciones y utilizados en la interacción cotidiana con los distintos miembros del grupo de pertenencia. En este sentido, un kimche, refiriéndose a los niños y jóvenes señala: "Sí, ellos saben de los apellidos de los tíos y de los primos, pero ahora la familia no se respeta. Al momento de saludar, lo hacen de lejos, no es como antes..." (S7, P135). En el testimonio se evidencia que el reconocimiento de los apellidos permite identificar los nexos parentales existentes entre los sujetos de la familia mapuche. Asimismo, devela una pérdida del respeto en la relación entre familiares, lo que se grafica en la práctica del saludo, que marca la distancia, coartando las posibilidades de una interacción más cercana y afectiva.

La importancia de este conocimiento parental radica en que los padres y principalmente los abuelos son un referente para la vida de los individuos. Quien proviene de una familia donde se reconoce positivamente la procedencia parental, trata de aprenderla y actuará en consecuencia. Asimismo, quien procede de una familia con características negativas, se comportará de esa misma forma. Un testimonio señala: "El küpan es lo más difícil de enseñar..., después de una generación, puede llegar un wewpife (orador). ... lo mismo puede pasar con una familia de logko (jefe)." (S6, P125). En el testimonio se devela que la historia del parentesco constituye un medio reconocido por la comunidad para llegar a ser kimche, cada cierta generación, lo que en mapunzugun se refiere al küpalme. El küpalme es el ejercicio de una herencia de saberes y conocimientos para la realización de un rol en la familia y la comunidad, lo que recae sobre un individuo que heredó el conocimiento familiar, según sus características. "Los Wechumpan, los Nawel, los Ayjapan, Kajfulikan... Ellos son descendientes de logko o genpiñ, por lo que están dirigiendo los gijatun, los elüwün, los machitun — ceremonias socio religiosas-... son las familias que dieron origen al lof (comunidad)" (S3, P159). En el testimonio, se constata la existencia de familias que heredan un conocimiento acerca de la realización de prácticas socioculturales y socio religiosas, lo que está en relación con el desarrollo de la historia local y el territorio Lafkenche. 
Uno de los conocimientos respecto del territorio que está presente en las comunidades mapuches es la asignación de nombres a los lugares. Los nombres que se les han otorgado a los distintos espacios están en relación con las características del territorio, como es el caso de la existencia de humedales y cerros. Ejemplo de esto es el caso del sector Cojülewfu, palabra que en mapunzugun significa río de color café; su color estaría en directa relación con el depósito de residuos de erosión provocada por la agricultura y la forestación. A continuación, un kimche señala otro ejemplo: "La comunidad de Rukaxaro, se llama así porque en ese lugar antes existían bosques nativos en los cuales habitaban muchas aves llamadas xarü (traro)... Los abuelitos decían y conversaban que por eso se nombra Rukaxaro" (S7, P17). En el testimonio se constata que la toponimia de lugares se relaciona con un conocimiento que permite interpretar y dar sentido a las características del medio natural.

Asimismo se considera la existencia de hitos referidos a espacios socio religiosos, correspondientes principalmente a los gijatuwe - lugar de realización de las ceremonias religiosas - . La importancia del gijatuwe radica en ser un espacio de reuniones sociales, culturales y espirituales. En la actualidad (generalmente) participa la totalidad de las familias por parte del padre y la madre. La totalidad de estos hechos están presentes en la memoria histórica de las familias y comunidades, siendo fundamentales para la educación familiar de niños y adolescentes.

\subsection{EDUCACIÓN FAMILIAR MAPUCHE}

Desde el discurso de los kimches se desprende que la educación familiar está compuesta por dos elementos fundamentales: las prácticas socioculturales mapuches y el conocimiento relativo a relaciones de parentesco. El primero está compuesto por tres categorías: 1) Prácticas socioculturales, referidas a la interacción entre personas de la misma o distinta comunidad. En este ámbito se identifican: a) el wixankontuwün, que se refiere a los actos de visita entre personas y familias, con el fin de conocer el estado de los otros en cuanto a su salud, hechos o sucesos que pudiesen estar viviendo y del avance de la vida. Por consiguiente, tiene como fin mantener las relaciones interpersonales y las alianzas establecidas entre los sujetos; $b$ ) el konchotun, que tiene la misma finalidad que el wixankontuwün, y se desarrolla mediante la entrega de obsequios de una familia a otra, siendo esto alternado entre los participantes, con el fin de asegurar un próximo encuentro entre los grupos (S2, P83); c) el lakutun, que es la unión de dos personas (un adulto y un niño) por medio de la asignación de un nombre en común, donde el adulto auxiliará las labores de formación y socialización de ese niño. 2) Prácticas socio religiosas, relacionadas con la interacción del ser humano con fuerzas espirituales donde se identifican: a) el gijatun, comprendido como una práctica socio religiosa que pone en relación temporo-espacial a las familias agrupadas en las comunidades y otras aledañas, con el fin de contactarse con las fuerzas espirituales por medio de rogativas fundamentadas en la memoria social, para mantener el equilibrio entre los distintos componentes del 
medio natural, social, cultural y espiritual (S1, P 112); b) el we xipantu, que es un espacio temporal en que se renueva el ciclo anual, comenzando uno nuevo. Es una época en que las familias se reúnen para agradecer a las fuerzas espirituales, para que el nuevo ciclo sea fructífero (S1, P165). 3) Un tercer elemento son las prácticas discursivas, las que constituyen un significativo recurso educativo para la formación de las nuevas generaciones. En este ámbito destacan dos tipos de discursos: a) el güxam, que es una práctica discursiva en forma de conversación en la que se enseñan y aprenden contenidos educativos, según las necesidades educativas inmediatas de los sujetos; y b) el ülkantun, que es un discurso poético expresado a modo de canción, en la que las personas expresan contenidos educativos y sus sentimientos, deseables para la formación de personas.

Un segundo componente de la educación familiar son los conocimientos relativos al parentesco, el que en su conjunto forma la identidad sociocultural de las nuevas generaciones y que tiene los siguientes componentes: a) el küpan como ascendencia familiar, referido a los conocimientos de los padres y abuelos, considerados estos últimos como la raíz parental y el conocimiento de los distintos integrantes del grupo parental y su nominación tradicional en mapunzugun (lengua mapuche). Esto con el fin de establecer relaciones fundamentadas en el respeto y evitar las uniones matrimoniales entre los miembros del grupo parental; b) el küpalme, referido a los roles sociales contenidos en la familia por generaciones, como lo es el caso de los logkos — máxima autoridad política de las comunidades—, y cuyo acceso a estos roles sociales depende de las fuerzas espirituales; y c) el tuwün como asentamiento familiar, relacionado con los conocimientos respecto de los hechos históricos desarrollados en este lugar, los saberes acerca de la geografía, los espacios en los que recaen representaciones culturales por ser habitados por fuerzas espirituales y la valoración del territorio. Por consiguiente, la educación familiar mapuche se sustenta en un conjunto de conocimientos construidos en la lógica propia y que aseguran la permanencia del estilo de vida y del patrimonio cultural mapuche.

\section{DISCUSIÓN Y CONCLUSIONES}

En general, en el discurso de los kimches se devela el hecho de estar directamente vinculado con los elementos territoriales y sobrenaturales que coexisten en el borde costero de La Araucanía. Estos elementos permiten la construcción de una cosmovisión propia que le otorga sentido a la identidad social, cultural y política Lafkenche, aun cuando estudios recientes muestran un bajo conocimiento de esta cosmovisión y del entorno natural inmediato de la escuela, en cuanto a características geográficas del territorio (Peña-Cortés, Gutiérrez, Rebolledo, Escalona, Hauenstein, Bertrán, Schlatter y Tapia, 2006; Almendra, Peña-Cortés y Rojas-Maturana, 2011). Sin embargo, en el contexto social, político y educacional actual, es cada vez más relevante considerar los elementos socioculturales y territoriales como base de 
conocimientos para contextualizar el currículum, desde un enfoque educativo intercultural.

En el borde costero se observan prácticas socioculturales relacionadas con un conjunto de acciones realizadas en las familias y comunidades, en las que se expresan sus saberes y conocimientos propios, permitiendo la proyección del patrimonio cultural mapuche en las nuevas generaciones. Sin embargo, de acuerdo con los testimonios de los kimches, en la actualidad la mayoría de los padres de familias han sido escolarizados en base a un currículum monocultural que históricamente ha suprimido los saberes mapuches.

En el contexto escolar se observa que las tensiones entre el conocimiento construido en la educación familiar y el conocimiento escolar son constantes. En general, los mapuches en la escuela aprenden el conocimiento disciplinario de las ciencias, pero no es independiente de la construcción social para comprender los sucesos naturales, la representación acerca del territorio y el sentido de su relación con la naturaleza. Paralelamente, aunque los kimches han sido escolarizados, conservan una sólida identidad sociocultural asociada a sus costumbres y los conocimientos parentales. Entonces, la relación de saberes y los procesos de cambios cognitivos en contexto mapuche no solo son abordables desde lo epistemológico, sino también desde el valor de las construcciones socioculturales que desarrollan los individuos para comprender contextos específicos.

En el contexto indígena, en Chile, la educación escolar es uno de los más amplios espacios de desarrollo de interacciones entre mapuches y no mapuches, incidiendo directamente en el desarrollo de las nuevas generaciones. Sin embargo, en este proceso la escuela no incorpora contenidos educativos o prácticas socioculturales validadas por la comunidad al currículum escolar. Así, la educación incide directamente en el desconocimiento de los miembros de la comunidad con respecto a los elementos de su propia cultura. Desde la perspectiva de los kimches, es deseable lograr un proceso de escolarización basado en la relación de saberes mapuches y no mapuches, ya que es fundamental para que las nuevas generaciones efectivamente interactúen en el contexto escolar, sin dejar de lado su propia identidad sociocultural. Este hecho permitiría al estudiante, por una parte, un proceso de reapropiación de la propia cultura por medio de la educación escolar, con el fin de evitar la pérdida de la identidad y del conocimiento educativo propio, asociado a saberes y prácticas socioculturales vigentes. Por otra parte, permitiría el aprendizaje del estudiante no mapuche respecto de saberes y conocimientos educativos mapuches, para transformarse progresivamente en un sujeto más intercultural.

Las prácticas socioculturales que se visualizan como elementos que permiten develar el conocimiento propio son: visitarse entre parientes, las prácticas socio religiosas, el conocimiento relativo al parentesco, el territorio, la historia local y el trabajo artesanal. En esta perspectiva, se plantea el desafío de la participación colaborativa entre la familia, la escuela y la comunidad como una estrategia posible 
para propiciar una educación intercultural, basada en la relación de saberes. Esto permitiría disminuir progresivamente la distancia entre la lógica del conocimiento mapuche y el escolar en la enseñanza de las ciencias. El objetivo sería contextualizar la enseñanza y el aprendizaje de las ciencias, sustentada en la aplicación de principios de enseñanza y aprendizaje fundados en la lógica del conocimiento mapuche e incorporarlos en protocolos de experimentación científica en aula.

En consecuencia, algunos de los principios que sustentan la enseñanza y el aprendizaje mapuche que permitirían contextualizar la educación escolar son: 1) Mirar y observar lo que se quiere aprender; 2) Escuchar la enseñanza de consejos o de conversaciones respecto de las características, procedimientos y naturaleza de los saberes vernáculos; 3) El conocimiento y reconocimiento de los recursos naturales, las capacidades de las personas para identificar, clasificar y categorizar elementos en una relación de respeto y armonía con el medio natural y espiritual; 4) La capacidad de desarrollar la obediencia y el respeto se relaciona con actividades que permiten al sujeto aprender, conversar y desarrollar el pensamiento, la memoria acerca de los diferentes procesos, como un saber y conocimiento educativo internalizado para el transcurso de la vida; y 5) La enseñanza y el aprendizaje colaborativo entre niños, jóvenes, adultos y ancianos como base de la acción educativa familiar que mantiene vivo y vigente los saberes y conocimientos mediante la oralidad en mapunzugun (véase Quilaqueo y Quintriqueo, 2010).

En conclusión, desde una epistemología contextual sugerimos la incorporación de los kimches (sabios) como mediadores para la enseñanza de las ciencias respecto de la identificación, clasificación y categorización de los vegetales asociado a sus usos en la vida natural, social, cultural y espiritual. Además, generar la relación de saberes en historia, geografia y ciencias sociales para estudiar y comprender la territorialidad, considerando los modos éticos de relacionarse con el medio natural y social, los que en su conjunto permitirían comprender de mejor forma la dinámica de los procesos sociales y naturales. Estas acciones educativas permitirían una valoración de los métodos de construcción del conocimiento mapuche y su aplicación en los protocolos experimentales de las ciencias. De este modo, se enriquecerían las habilidades del pensamiento científico y la valoración 'del otro como un legítimo otro' entre estudiantes mapuches y no mapuches, que de algún modo están implicados en la construcción de un diálogo intercultural.

Universidad Católica de Temuco* Centro de Investigación en Educación en Contexto Indígena e Intercultural Casilla 15-D, Tетисо (Chile) squintri@uct.cl 
Segundo Quintriqueo, Daniel Quilaqueo, Fernando Peña-Cortés y Gerardo Muñoz

Universidad Católica de Temuco**

Facultad de Educación

Casilla 15-D, Teтuco (Chile)

dquilaq@uct.cl

Universidad Católica de Temuco***

Casilla 15-D, Tетисо (Chile)

fpena@uct.cl.

Univertitat Autónoma de Barcelona ${ }^{* * * *}$

Cerdanyola del Vallés, Barcelona (España)

geramaster@gmail.com

\section{OBRAS CITADAS}

Almendra, Osvaldo, Fernando, Peña-Cortés y Marcela, Rojas-Maturana. Relación entre saberes y conocimientos territoriales en escuelas Lafkenches: La necesidad de un currículum intercultural con base geográfica. REXE 10(20): 32-68, 2011.

Alsina, Miquel. Comunicación intercultural. Barcelona: Anthropos Editorial, 1999.

Ardoino, Jacques. Complejidad y formación. Pensar la educación desde una mirada epistemológica. Buenos Aires: Novedades Educativas, 2005.

Beillerot, Jacky y Mosconi, Nicole. Traité des sciences et des pratiques de l'éducation. Paris: Dunod, 2006.

Bisquerra, Rafael. Metodología de la investigación educativa. Madrid: La Muralla, 2004.

Baudelot, Chistrian y Leclerq, François. Efectos de la educación. Buenos Aires: Del estante editorial, 2008.

Bonal, Xavier. Sociología de la educación: Una aproximación crítica a las corrientes contemporáneas. Barcelona: Paidós, 1998.

Castellano, Ana y Hinestroza, Jorge. Una perspectiva epistemológica para el estudio de las formaciones socioculturales. Revista de Ciencias Sociales, Vol. XV, $\mathrm{N}^{\circ}$ 4, Octubre-Diciembre, 2009: 681-692.

Cyrulnik, Boris y Morin, Edgar. Diálogos sobre la naturaleza humana. Barcelona: Paidós, 2005.

Durán, Teresa y Cárdenas, Prosperino. Naturaleza social de la Educación Básica chilena. Configuración e inserción del sistema. Santiago: Pontificia Universidad Católica de Chile, Departamento de Educación, 1983.

Egaña, María. La educación primaria popular en el siglo XIX en Chile: Una práctica de política estatal. Santiago: LOM Ediciones, 2000. 
Filippa, Nelly y Schiattino, Elizabeth. "La transposición del conocimiento en clases". En Brandi, L. Conocimiento escolar y cultura institucional. Madrid: Miño y Dávila, 1997.

Fourez, Gérard. Apprivoiser l'épistemologie. Bruxelles: Édition De Boeck Université, 2009.

García, Josefa. El conocimiento y el currículum en la escuela: El reto de la complejidad. Buenos Aires: Homo Sapiens, 2007.

Gauthier, Clermont, Desbiens, Jean-François, Malo, Annie, Martineau, Stéphane y Simard, Denis. Pour un Théorie de la Pédagogie. Recherches Contemporaines sur le savoir de enseingants. Québec: Le Presses de l'Université Laval, 1997.

Gauthier, Benoît. Recherche sociale. De la problématique à la collecte des données. Tse-Foy: Presses de l'Université du Québec, 2009.

Izquierdo, Adolfo. Espacio-temporalidad y omnijetividad. Una aproximación Epistemológica. En: Revista Nómadas, $\mathrm{N}^{\circ}$ 11. Universidad de los Andes. Cider. Bogotá, 1998: 241-247.

Jamioy, José. Los saberes indígenas son patrimonio de la humanidad. Revista Nómada, $\mathrm{N}^{\circ}$ 7, 1997: 64-72.

Krippendorff, Klaus. Metodología de análisis de contenido. Teoría y práctica. Barcelona: Paidós, 1990.

López, María. Merleau-Ponty: Imbricación en el mundo con los otros. En Revista de Filosofía, $\mathrm{N}^{\circ}$ 44, 2008: 173-184.

Mutuale, Agustin. Bernard Charlot y la práctica del saber. EDUCERE, Año 13, N 44, Enero-Febrero-Marzo, 2009: 227-233.

Nadeau, Robert. Philosophies de la connaisance. Québec: Les Presses de l'Université Laval, 2009.

Olivé, León. "Por una auténtica interculturalidad basada en el reconocimiento de la pluralidad epistemológica". En Olivé, L. et al. Pluralismo Epistemológico. La Paz, Bolivia: Muela del Diablo Editores, 2009: 191-218.

Peña-Cortés, Fernando, Almendra, Osvaldo y Rojas, Marcela. "Enfoque geográfico territorial de la educación intercultural". En Quilaqueo, D., Fernández, C. y Quintriqueo, S. Interculturalidad en contexto mapuche. Neuquén: Educo, 2010: 241-256.

Peña-Cortés, Fernando, Gutiérrez, Patricia, Rebolledo, Gonzalo, Escalona, Miguel, Hauenstein, Enrique, Bertrán, Carlos, Schlatter, Roberto y Tapia, Jaime. Determinación del nivel de antropización de humedales como criterio para la planificación ecológica de la cuenca del lago Budi, IX Región de La Araucanía, Chile. Revista Geográfica Norte Grande, № 36, 2006: 75-91.

Pérez, Gloria. Investigación cualitativa. Retos e interrogante. Madrid: Colección Aulas Abiertas, 2000.

Quilaqueo, Daniel, Fernández, César y Quintriqueo, Segundo. Interculturalidad en contexto mapuche. Neuquén: Educo, 2010. 
Quilaqueo, Daniel y Quintriqueo, Segundo. Saberes educativos mapuches: un análisis desde la perspectiva de los kimches. Revista Latinoamericana POLIS, V. 9, N²6, 2010: 337-360.

Quintriqueo, Segundo, Quilaqueo, Daniel y Torres, Héctor. Contribución para la enseñanza de las ciencias naturales: saber mapuche y escolar. Educação e Pesquisa. Vol. $40 \mathrm{~N}^{\circ}$ 4, São Paulo, Oct./Dec. 2014

Quintriqueo, Segundo y Torres, Héctor. Construcción de conocimiento mapuche y su relación con el conocimiento escolar. Revista Estudios Pedagógicos, XXXIX(1), 2013: 199-216.

Quintriqueo, Segundo y Torres, Héctor. Distancia entre el conocimiento mapuche y el conocimiento escolar en contexto mapuche. Revista Electrónica de Investigación Educativa, 14(1), 15-31, 2012. Disponible en:

http://redie.uabc.mx/vol14nol/contenido-quintriqueotorres.html

Quintriqueo, Segundo. Implicancias de un modelo curricular monocultural en contexto mapuche. Santiago de Chile: Gráfica LOM, 2010.

Quintriqueo, Segundo y Cárdenas, Prosperino. "Educación Intercultural en contextos mapuches. Articulación entre el conocimiento mapuche y el occidental en el ámbito de las ciencias". En Quilaqueo, D., Fernández, C. y Quintriqueo, S. (2010). Interculturalidad en contexto mapuche. Neuquén: Educo, 2010.

Quintriqueo, Segundo y McGinity, Margaret. Implicancias de un modelo curricular monocultural en la construcción de la identidad sociocultural de alumnos/as mapuches de la IX Región de La Araucanía, Chile. Estudios Pedagógicos, №2, Valdivia, 2009: 173-188.

Quivy, Raymond y Campenhoudt, Luc. Manual de Investigación en Ciencias Sociales. México: Edición Limusa, 1998.

Ramírez, Ángel. Problemas teóricos del conocimiento indígena. Presupuestos e inquietudes epistemológicas de base. Revista Yachaikuna, № 1, marzo, 2001.

Rockwell, Elsie. Hacer escuela, hacer estado. La educación posrevolucionaria vista desde Tlaxcala, México: Colegio de Michoacán, Centro de Investigaciones y Estudios Superiores en Antropología Social (CIESAS) y Centro de Investigación y de Estudios Avanzados (Cinvestav), 2008.

—L La escuela cotidiana. México: Fondo de Cultura Económica, 1995.

Rodrigo, María y Arnay José. La construcción del conocimiento escolar. Barcelona: Ediciones Paidós, 1997.

Ruiz, Carlo. El Enfoque Multimétodo en la Investigación Social y Educativa: Una Mirada desde el Paradigma de la Complejidad. Revista de Filosofia y Sociopolítica de la Educación. № 8. Venezuela, 2008.

Tashakkori, Abbas y Teddlie, Charles. Handbook of mixed social and behavioral research. London: Sage, 2003. 